\title{
Frequência de parasitos intestinais, em amostras de fezes de cães e gatos, recebidas no laboratório de doenças parasitarias da UFPel, entre os anos de 2015 e 2016
}

\author{
[Frequency of intestinal parasites, in stool samples of dogs and cats, received in the laboratory of \\ parasitary diseases of UFPel, between the years 2015 and 2016]
}

\section{"Artigo Científico/Scientific Article"}

\author{
Alexsander Ferraz ${ }^{1 *}$, Bruna dos Santos Pires $^{1}$, Eduarda Machado dos Santos ${ }^{1}$, \\ Tainá Ança Evaristo ${ }^{1}$, Bruno Cabral Chagas ${ }^{2}$, Ana Lúcia Coelho Recuero ${ }^{1}$, \\ Tanize Angonesi de Castro ${ }^{1}$, Leandro Quintana Nizoli ${ }^{1}$
}

\author{
${ }^{1}$ Departamento de Veterinária Preventiva, Universidade Federal de Pelotas (UFPel), Pelotas-RS, Brasil. \\ ${ }^{2}$ Departamento de de Microbiologia e Parasitologia, Universidade Federal de Pelotas (UFPel), Pelotas-RS, Brasil. \\ *Autor para correspondência/Corresponding author: E-mail: xanderferraz@yahoo.com.br
}

\begin{abstract}
Resumo
Objetivou-se determinar a frequência de helmintos gastrintestinais de cães e gatos, em amostras fecais, recebidas e analisadas do laboratório de doenças parasitárias (Ladopar) da Faculdade de Veterinária, UFPel. No período de 2015 a 2016, foram examinadas, 737 amostras fecais, sendo 709 de cães e 28 de gatos. Os animais avaliados, pertenciam a ambos os sexos, idades variadas e diferentes raças. As fezes foram processadas pelo método de Willis \& Mollay (1921). Dentre as amostras avaliadas, $470(63,77 \%)$ apresentaram-se positivas para algum gênero de parasito. Do total das amostras positivas, $301(64 \%)$ apresentaram infecção única, enquanto que 169 (36\%) apresentaram coinfecção por parasitos gastrointestinais. O gênero, encontrado em maior frequência, em ambas espécies, foi o Ancylostoma spp. representando 52,04\% das amostras dos cães e 17,86\% dos gatos. A ocorrência de parasitos com potencial zoonótico e o estreito contato entre os animais e o homem mostra a necessidade de um controle efetivo e específico, visto que a redução da carga parasitária dos animais e consequentemente do ambiente, diminui a exposição dos humanos a importantes zoonoses.
\end{abstract}

Palavras-chave: helmintos; zoonoses; Ancylostoma; diagnóstico.

\begin{abstract}
The objective of this article was to determine the frequency of gastrointestinal helminths of dogs and cats in faecal samples collected and analyzed from the Laboratory of Parasitic Diseases (Ladopar) of the Faculty of Veterinary Medicine, Federal University of Pelotas/UFPel, Brazil. In the period between 2015 and 2016, 737 fecal samples were examined, with 709 from dogs and 28 from cats. The evaluated animals belonged to both sexes, varied ages and different breeds. The faeces were processed by the Willis \& Mollay method (1921). Among the samples evaluated, $470(63.77 \%)$ were positive for some genus of parasite, being that, from the total of the positive samples, 301 (64\%) presented a single infection, while $169(36 \%)$ presented co-infection by gastrointestinal parasites. The genus most frequently found in both species was Ancylostoma spp., representing $52.04 \%$ of the samples from the dogs and $17.86 \%$ from the cats. The occurrence of parasites with zoonotic potential and the close contact between animals and humans show the need for effective and specific control, since the reduction of the parasitic load of the animals and consequently of the environment reduces the exposure of humans to important zoonosis.
\end{abstract}

Keywords: helminths; zoonosis; Ancylostoma; diagnosis.

\section{Introdução}

O convívio entre pessoas e animais domésticos, principalmente, cães, gatos e equinos promovem uma melhora social, emocional e

cognitiva para o ser humano (Machado et al., 2008). 
Pelo fato, do cão e gato serem as espécies, que mantém maior contato físico com o homem, o controle sanitário destes animais, se faz, de extrema importância. Dentre várias doenças que acometem os cães e gatos, as helmintoses merecem atenção e destaque na clínica veterinária e na saúde pública (Acha e Szyfres, 2003). Segundo Oliveira et al. (2009) e Campos Filho et al. (2008), o estreitamento entre as relações estabelecidas entre humanos e animais, facilita a disseminação de parasitos causadores de doenças parasitárias potencialmente zoonóticas, podendo, estes animais, contaminarem o ambiente onde convivem, através da liberação de ovos de diversos gêneros de parasitos, nas fezes.

A falta de manejo adequado no ambiente e tratamento periódico dos animais, estão relacionadas diretamente com o modo em que a disseminação parasitária pode ocorrer (Souza, et al., 2002). Ancylostoma spp., são parasitos gastrointestinais de cães e gatos que podem causar erosão de mucosa intestinal, diarreia apresentando sangue, levando a anemia severa, dependendo do grau de infecção. No homem, esses parasitos causam inflamações cutâneas, com erupções serpinginosas na pele, denominada larva migrans cutânea (LMC), ou popularmente conhecida como bicho-geográfico (Andrade et al., 2012).

Toxocara sp., outro parasito zoonótico gastrintestinal de cães e gatos, é frequente em áreas urbanas, ocorrendo maior disseminação em locais onde há menor saneamento básico e maior quantidade de animais jovens (Lima, 2011). A infecção em humanos por Toxocara sp. acontece de forma acidental, levando ao quadro clínico denominado larva migrans visceral (LMV), podendo, em casos mais graves, após migrações no organismo acarretar larva migrans ocular (LMO) (Rubinsky-Elefant et al., 2010)

Trichuris sp. é um helminto presente no ceco e cólon de cães e outros canídeos silvestres, as fontes de infecção são o solo e os cursos de águas contaminados com os ovos do parasita. Nos animais com alta infestação, ocorre dor, distensão abdominal, juntamente com diarreia. Segundo Acha e Szyfes (2003), em animais muito jovens, principalmente aqueles com imunidade baixa e com grande carga parasitária, pode ocorrer prolapso retal.

Também podemos citar, o cestódeo Dipylidium caninum, um parasito habitual do intestino delgado de cães e que esporadicamente pode infectar o homem, tendo como hospedeiro intermediário a pulga Pulex irritans, Ctenocephalides canis, Ctenocephalides felis e o piolho Trichodectes canis (Wani et al., 2013). Seus ovos são eliminados nas fezes, dentro da cápsula ovígera, podendo conter aproximadamente 30 ovos (Taylor et al. 2014) e o hospedeiro definitivo infecta-se através da ingestão da pulga ou do piolho contendo os cisticercoides (Robertson e Thompson, 2002).

Este estudo teve como objetivo, avaliar a frequência dos principais parasitos gastrintestinais de cães e gatos diagnosticados no Laboratório de Doenças Parasitárias (Ladopar) da Faculdade de Veterinária da UFPel.

\section{Material e métodos}

Amostras de fezes de cães e de gatos foram recebidas no laboratório de doenças parasitárias (Ladopar), da Faculdade de Veterinária da UFPel, entre janeiro de 2015 e dezembro de 2016, totalizando 737 amostras, destas, 709 eram de cães (96\%) e 28 de gatos (4\%). As amostras analisadas eram provenientes de animais de ambos os sexos, idades variadas e diferentes raças.

As amostras analisadas chegaram ao Ladopar acondicionadas em recipientes plásticos, com gelo biológico, devidamente identificadas e imediatamente realizada a análise coproparasitológica.

O método de diagnóstico utilizado para pesquisa de ovos de helmintos e oocistos de protozoários foi a técnica de Willis-Mollay (1921) modificada.

\section{Resultados}

Do total de 737 amostras analisadas, 470 foram positivas para pelo ao menos um gênero de parasito intestinal, representando $63,77 \%$ das amostras. Das amostras positivas, 301 (64\%) apresentavam infecção simples e 169 (36\%) coinfecção.

$\mathrm{O}$ parasito encontrado em maior frequência nas fezes de cães e gatos, foi Ancylostoma spp., (Tabela 1).

A principal associação parasitária encontrada, foi de Ancylostoma/Trichuris e Ancylostoma/Toxocara, encontradas em 95 e 51 amostras, respectivamente, todas de cães (Tabela 2), em gatos foi observado somente infecção simples. 
Tabela 1. Frequência de parasitos, encontrados em amostras fecais de cães e gatos, analisadas no laboratório de doenças parasitárias da Faculdade de Medicina Veterinária da UFPel, no período de janeiro de 2015 a dezembro de 2016. Pelotas - RS.

\begin{tabular}{|c|c|c|c|}
\hline Espécie & Frequência & $\%$ positivos $(458)$ & $\%$ total (709) \\
\hline \multicolumn{4}{|l|}{ Cães $(\mathrm{n}=709)$} \\
\hline Ancylostoma spp. & 369 & 80,57 & 52,04 \\
\hline Toxocara sp. & 78 & 17,03 & 11,00 \\
\hline Trichuris sp. & 139 & 30,35 & 19,60 \\
\hline Toxascaris spp. & 4 & 0,87 & 0,56 \\
\hline Dipylidium caninum & 15 & 3,27 & 2,11 \\
\hline \multirow[t]{2}{*}{ Cystoisospora sp. } & 7 & 1,53 & 0,99 \\
\hline & Frequência & \% positivos (12) & $\%$ total (28) \\
\hline \multicolumn{4}{|l|}{ Gatos $(n=28)$} \\
\hline Ancylostoma spp. & 5 & 41,67 & 17,86 \\
\hline Toxocara sp. & 5 & 41,67 & 17,86 \\
\hline Cystoisospora sp. & 2 & 16,66 & 7,15 \\
\hline
\end{tabular}

Tabela 2. Frequência de coinfecção nas amostras fecais de cães, analisadas no Ladopar, entre janeiro de 2015 e dezembro de 2016.

Parasitos

Total Coinfecção (169)

\begin{tabular}{|c|c|c|}
\hline Ancylostoma spp. + Trichuris sp. & 95 & 56,2 \\
\hline Ancylostoma spp. + Toxocara sp. & 51 & 30,2 \\
\hline $\begin{array}{l}\text { Ancylostoma spp. + Toxocara sp. } \\
+ \text { Trichuris sp. }\end{array}$ & 11 & 6,5 \\
\hline Ancylostoma spp. + Toxascaris spp. & 4 & 2,4 \\
\hline Ancylostoma + Dipilydiun caninum & 3 & 1,8 \\
\hline Trichuris sp. + Dipylidium caninum & 1 & 0,6 \\
\hline Toxocara sp. + Dipylidium caninum & 1 & 0,6 \\
\hline $\begin{array}{l}\text { Ancylostoma spp. + Dipylidium caninum } \\
+ \text { Toxascaris spp. }\end{array}$ & 1 & 0,6 \\
\hline $\begin{array}{l}\text { Ancylostoma spp. + Trichuris sp. } \\
+ \text { Toxascaris spp. }\end{array}$ & 1 & 0,6 \\
\hline $\begin{array}{l}\text { Ancylostoma spp. + Trichuris sp. + Dipylidium } \\
\text { caninum }\end{array}$ & 1 & 0,6 \\
\hline Total & 169 & 100 \\
\hline
\end{tabular}

\section{Discussão}

No presente estudo, verificou-se que $63,77 \%$ das amostras analisadas estavam parasitadas, percentual mais baixo ao encontrado por Silva et al. (2007), que encontraram $87,9 \%$ de positividade, avaliando 240 amostras, no município de Santa Maria, RS.

O parasito encontrado em maior frequência, nas amostras processadas, foi do gênero Ancylostoma spp., responsável pela larva migrans cutânea. Scaini, et al. (2003) avaliando a contaminação ambiental por ovos de helmintos no balneário Cassino, RS, encontraram, uma frequência de $71,3 \%$ para Ancylostoma spp. nas amostras analisadas.

Diferentemente do encontrado no presente estudo, Ferreira et al. (2013), encontraram, em amostras de cães e gatos, uma frequência de 8,82\% (2290) para cães e 9,26\% (378) para gatos, percentual mais baixo que o encontrado neste estudo.

As várias formas de contaminação, podem explicar, porque a infecção por ancilostomídeos, nos cães e gatos, é maior, do que os outros parasitos. Dentre estas formas, é possível citar, ingestão de larvas de A. caninum, penetração de larvas na pele e também a transmissão de larvas de Ancylostoma através do leite materno (Chandler et al., 1989).

As infecções por Trichuris sp., apresentaram a segunda maior ocorrência nas amostras analisadas, com 19,6\% de positividade, diferindo de Leite et al. (2006), que observaram uma 
frequência de $2 \%$ deste parasito, analisando 150 amostras de fezes de cães, em Itapema, SC, estes valores são menores do que os encontrados neste estudo.

Resultado semelhante ao presente estudo, foi encontrado por Bresciani et al. (2008), que após analisar a carga parasitária de cães do município de Araçatuba, SP, verificou que $20 \%$ das 65 amostras eram positivas para Trichuris sp.

A frequência de Toxocara sp. foi de $11 \%$ em cães e $17,9 \%$ em gatos, valores semelhantes aos encontrados por Leite et al. (2007), que analisaram a ocorrência de parasitos, nas fezes de 280 cães e 30 gatos, na região metropolitana de Curitiba PR.

A elevada frequência de helmintos nas amostras analisadas, principalmente dos gêneros Ancylostoma e Toxocara, que além de serem patogênicos para os animais possuem potencial zoonótico, indicam a necessidade de medidas para a conscientização da população, controle e prevenção dessas enfermidades parasitárias (Bresciani et al., 2008).

\section{Conclusão}

Os resultados encontrados neste levantamento, indicam que há a necessidade de desenvolvimento de medidas estratégicas de profilaxia contra parasitos gastrintestinais de cães e gatos com potencial zoonótico, incluindo educação sanitária junto aos tutores de animais no município de Pelotas, RS.

\section{Conflito de Interesse}

Os autores declaram não existir conflito de interesse.

\section{Comitê de Ética}

Não houve necessidade de aprovação pelo comitê de ética, visto que a metodologia do trabalho baseou-se em um levantamento de amostras de fezes recebidas no laboratório para diagnóstico parasitológico.

\section{Referências}

Acha, P.N; Szyfres, B. Zoonosis y enfermidades transmisibles comunes al hombre ya los animales. Revista Espanõla de Salud Pública, 75(3): 263-264, 2003.

Andrade, V.A.; Costa, M.A.F.; Barbosa, J.V. Ocorrência de ovos de Ancylostoma spp. em amostras de fezes de gatos (Felis catus LINNAEUS, 1758) domiciliados em uma área escolar da Região Metropolitana do Rio de
Janeiro, RJ, Brasil. Cadernos UniFOA, 7(20): 115-123, 2012.

Bresciani, K.D.S.; Ishizaki, M.N.; Kaneto, C.N.; Montana, T.R.P.; Perri, S.H.V.; Vasconcelos, R.O.; Nascimento, A.A. Frequência e intensidade parasitária de helmintos gastrintestinais em cães na área urbana do município de Araçatuba, SP. Ars Veterinária, 24(3): 181-185, 2008.

Campos filho, P.C.; Barros, L.M.; Campos, J.O.; Braga, V.B.; Cazorla, I. M.; Albuquerque, G. R.; Carvalho, S. M. S. Parasitas zoonóticos em fezes de cães em praças públicas do município de Itabuna, Bahia, Brasil. Revista Brasileira de Parasitologia Veterinária, 17(4): 206209, 2008.

Chandler, E.A.; Hilbery, A.D.R.; Gaskell, C.J. Medicina e terapêutica de felinos. $2^{\mathrm{a}}$ ed. São Paulo: Manole, 1989. p. 359.

Ferreira, F.P.; Dias, R.C.F.; Martins, T.A.; Constantino, C.; Pasquali, A.K.S.; Vidotto, O.; Freire, R.L.; Navarro, I.T. Frequência de parasitas gastrointestinais em cães e gatos do município de Londrina, PR, com enfoque em saúde pública. Semina: Ciências Agrárias, 34(6), supl.2: 3851-3858, 2013.

Leite, L.C.; Bandeira, C.R.; Cirio, S.M.; Luz, E.; Diniz, J.M.F.; Leite, S.C.; Lunelli, D.; Weber, S.; Coelli, C.R.V.R. Ocorrência de ovos de Ancylostoma spp e Trichuris spp em fezes de cães em meia-praia, Itapema, Santa Catarina, Brasil. Revista Estudos de Biologia, 28(65): 105-110, 2006.

Leite, L.C.; Círio, S.M.; Silva, M.A.N.; Zadorosnei, A.C.B.; Luz, E.; Marinoni, L.P.; Leite, S.C.; Lunelli, D. Ocorrência de endoparasitas em amostras de fezes de cães (Canis familiaris) da região metropolitana de Curitiba, Paraná - Brasil. Revista Estudos de Biologia, 29(68/69): 319-326, 2007.

Lima, W.S. Larva migrans. In: Neves, D.P. Parasitologia humana. São Paulo: Atheneu, 2011. 291-294 p.

Machado, J.A.C.; Rocha, J.R.; Santos, L.M.; Piccinin, A. Terapia assistida por animais (TAA). Revista Científica Eletrônica de Medicina Veterinária, 6(10): 1-7, 2008.

Oliveira, S.F.O.; Melo, D.P.G.; Fernandes, P.R.; Schulze, C.M.B.; Guimarães, M.S.; Silva, Q.C. Ocorrência de helmintos gastrintestinais em cães errantes da cidade de Goiânia - Goiás. Revista de Patologia Tropical, 38(4): 279283, 2009. 
Robertson, I.D.; Thompson, R.C. Enteric parasitic zoonoses of domesticated dogs and cats. Microbes and Infection, 4(8): 867-873, 2002

Rubinsky-Elefant, G.; Hirata, C.E.; Yamamoto, J.H.; Ferreira, M.U. Human toxocariasis: diagnosis, worldwide seroprevalences and clinical expression of the systemic and ocular forms. Annals of Tropical Medicine and Parasitology, 104(1): 3-23, 2010.

Scaini, C.J.; Toledo, R.N.; Lovatel, R.; Dionello, M.A.; Gatti, F.A.; Susin, L. Contaminação ambiental por ovos e larvas de helmintos em fezes de cães na área central do Balneário Cassino, Rio Grande do Sul. Revista Sociedade Brasileira de Medicina Tropical, 36(5): 617-19, 2003.

Silva, A.S.; Ceolin, L.V.; Cargnelutti, J.F.; Pessoa, G.A.; Oliveira, C.B.; Neto, A.P.; Monteiro, S.G. Prevalência de parasitismo em cães domiciliados num bairro de Santa Maria RS. Saúde, 33(1): 27-31, 2007.
Souza, L.C., Modolo, J.R.; Padovani, C.R., Mendonça, A.O., Lopes, A.L.S., Silva, W.B. Posse responsável de cães no município de Botucatu- SP: realidades desafios. Revista de Educação Continuada do Conselho Regional de Medicina Veterinária, 5(2): 226-232, 2002.

Taylor, M.A.; Coop, R.L.; Wall, R.L. Parasitas de cães e gatos. Parasitologia Veterinária. 3 . ed. Rio de Janeiro: Guanabara Koogan, 2016, Cap. 6.

Urquhart, G.M.; Armour, J.; Duncan, J.L.; Dunn, A.M.; Jennigs, F.W. Parasitologia Veterinária, 2. ed. São Paulo: Guanabara Koogan, 1998.

Wani, Z., Allaie, I., Shah, B., Raies, A., Athar, H; Junaid, S. Dipylidium caninum infection in dogs infested with fleas. Journal of Parasitic Diseases, 39(1): 73-75, 2013.

Willis, I. I. A simple levitation method for the detection of hookworm ova. Medical Journal of Austrália, 2(18): 375-376, 1921. 